\title{
Size-Dependent RIS and FOM of Ag-Fe and Au-Fe Bimetallic Alloys in Triangular Prism: A DDA Study
}

\author{
Pradeep BHATIA*, S. S. VERMA, and M. M. SINHA \\ Department of Physics, Sant Longowal Institute of Engineering and Technology, Sangrur-148106, Punjab, India \\ *Corresponding author: Pradeep BHATIAＥmail: pardeepbhatiahp@gmail.com
}

\begin{abstract}
In this paper, the localized surface plasmon resonance (LSPR) peak of Ag-Fe and Au-Fe alloy nanoparticles for the triangular prism is calculated by using the discrete dipole approximation (DDA) method. We investigate the variation of the resonance wavelength, refractive index sensitivity, and figure of merit with the particles size and alloy compositions. We perform a comparative study on the refractive index sensitivity and figure of merit of alloys in order to find the considered (Ag-Fe and $\mathrm{Au}-\mathrm{Fe}$ ) alloys with high sensitivity. The refractive index sensitivity of the Au-Fe alloy is found higher than that of the Ag-Fe alloy. Therefore, to optimize the size of alloy nanoparticles (NPs) for the triangular prism, the figure of merit is calculated and observed that the optimized size is $50 \mathrm{~nm}$ and $20 \mathrm{~nm}$ for $\mathrm{Ag}-\mathrm{Fe}$ and $\mathrm{Au}-\mathrm{Fe}$ alloys, respectively. A comparison of Ag-Fe shows that the Au-Fe alloy NPs have greater figure of merit (FOM) and thus may be more suitable for applications in biosensing.
\end{abstract}

Keywords: Nanoparticles; alloys; refractive index sensitivity; figure of merit

Citation: Pradeep BHATIA, S. S. VERMA, and M. M. SINHA, "Size-Dependent RIS and FOM of Ag-Fe and Au-Fe Bimetallic Alloys in Triangular Prism: A DDA Study," Photonic Sensors, 2019, 9(3): 246-258.

\section{Introduction}

The current status of research takes account of the combination of magnetism with plasmonic materials or nanoparticles (NPs) towards catalysis, biosensing, and drug delivery applications. NPs from the group of transition metals are of great interest due to their beneficial properties such as magnetic moment and coercivity in comparison with the bulk counterpart and high specific surface area [1]. From the transition metal group, the magnetic NPs have promising practical applications such as catalysis, magnetic recording media, and magnetic fluids [2]. But the magnetic nanoparticles (MNPs) get easily oxidized in air or moisture, which limits their potential biomedical uses.
Several approaches have been investigated to overcome this limitation. The most effective and widely used approach is the coating of MNPs with the protective shell (core-shell). Another approach is the mixing of two or more metallic NPs as alloys formation. Most importantly, core-shell and alloy nanostructures not only resist the oxidation and magnetic properties but also increase their stability and functionality. Such multifunctional nanoparticles have a great potential for optic-electronic devices, diagnostic, therapeutic, and biomedical devices. Various synthesis routes of core-shell and alloys nanostructures have been well established theoretically as well as experimentally [3, 4]. V. Amendola et al. synthesized Fe-Ag NPs in one step by pulsed laser

Received: 25 October 2018 / Revised: 12 January 2019

(C) The Author(s) 2019. This article is published with open access at Springerlink.com DOI: $10.1007 / \mathrm{s} 13320-019-0547-8$ 
ablation, which were studied by using the discrete dipole approximation (DDA) method of numerical calculations based on the theoretical approach [5]. The control of thickness, either on the core or on the shell, allows the tuning of plasmonic properties of the core-shell NPs in the visible to near infra-red (NIR) wavelengths of the electromagnetic (EM) spectrum [6].

Many researchers studied the plasmonic properties of noble metals for sensors based on the localized surface plasmon resonance (LSPR) [7, 8]. J. Katyal et al. studied the plasmonic properties of $\mathrm{Al}$ nanospheres, nanorods, and nanodisks for sensing [9], and their results showed that $\mathrm{Al}$ is an efficient plasmonic material for refractive index sensing in ultra-violet (UV) to NIR wavelengths. G. H. Chan et al. studied the LSPR spectroscopy of triangular aluminum (Al) nanoparticles [10]. The LSPR spectroscopy depends on the size, shape, compositions, core-shell thickness, as well as the particles spacing [11-14], and it is sensitive to the changes in the surrounding medium which attracts much attention towards metal NPs. Due to the enhancement of the electromagnetic field near the metallic nanostructure, the surface plasmon resonance (SPR) based sensors are immensely sensitive to the change in the refractive index of the the metal surface. There are different detection methods of SPR sensors such as the wavelength, angle, intensity, and phase interrogations, in which wavelength interrogation method has great advantages over the other methods of SPR sensors, such as multi-channel and multi-parameter measurement. There are many applications of SPR sensors in various fields such as biosensing, drug selection, clinical diagnosis, food detection, and environmental monitoring [15-17].

When a small spherical metallic nanoparticle is irradiated by light, the oscillating electric field causes the conduction electrons to oscillate coherently. The oscillation frequency is determined by four aspects: the density of electrons, the effective electron mass, and the shape and size of the charge distribution. The important property of noble metals, i.e., resonant oscillation of conduction electrons at the interface between the noble metal and dielectric, is called SPR. Recent studies on metallic nanoparticles of either spherical or non-spherical shape have a growing interest due to their intense, valuable, and distinct optical properties which result in new approaches in sensing and imaging applications at extremely low detection limits [18]. Further, the ability to provide a large electric field enhancement with triangular shaped NPs makes them as an attractive option for the application in sensing. Many researchers [19-22] have described the suitable synthesis approach of bimetallic Fe-Ag NPs as core-shell and alloys by pulsed laser ablation in a different liquid solution. Different surrounding media are the important challenge to achieve the compatibility within biological samples without harming the specimens. Thus, LSPR spectra can also be improved by adjusting the refractive index of the environment. All of these factors motivate researchers to investigate the primary optical properties such as extinction, absorption, and scattering of bimetallic alloy nanoparticles of varying shapes and sizes in different surrounding media.

The optical properties of the triangular prism $\mathrm{Ag}-\mathrm{Fe}$ and $\mathrm{Au}-\mathrm{Fe}$ bimetallic alloy system with different surrounding environments have not been explored. In triangular-shaped geometry of nanoparticles, the number of corners attributes to the multipolar mode due to high polarization. The appearance of multipolar plasmon modes may be seen as the retardation effect depending on the relative size of NPs, however, the considered triangular-prism (1:1:2) may exhibit interesting sensing properties. Therefore, we propose a triangular prism nanostructure of $\mathrm{Ag}-\mathrm{Fe}$ and $\mathrm{Au}-\mathrm{Fe}$ nanoparticles with $20 \mathrm{~nm}$ to $70 \mathrm{~nm}$ particles size in different surrounding media, which is depicted in Fig. 1. The discrete dipole approximation is used to 
calculate the absorption efficiency and optical resonance wavelength of bimetallic alloy nanoparticles. The preliminary interesting optical properties shown by the Au-Fe triangular prism and reported in [23] have motivated us to study further the mechanism of LSPR for sensing of magneto-plasmonics NPs of iron alloys with Au and Ag. Furthermore, we have calculated the refractive index sensitivity (RIS) and figure of merit (FOM) of $\mathrm{Ag}-\mathrm{Fe}$ and $\mathrm{Au}-\mathrm{Fe}$ alloy nanoparticles and made a comparison of two considered bimetallic systems.

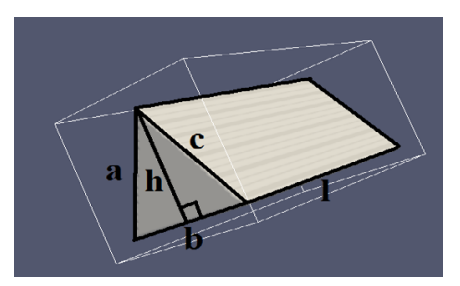

Fig. 1 Illustration of the triangular prism nanostructure.

\section{Computational method}

There are various numerical methods like DDA, finite difference time domain (FDTD), and finite element method (FEM) to calculate the optical properties such as absorption, scattering, and extinction [24-26] of nanostructures. DDA is one of the most powerful, flexible electro-dynamic numerical methods and freely available to investigate the optical properties for targeted nanostructures of any arbitrary geometry. In DDA, the target geometry is described as an array of number $N$ of dipoles points [27]. The position or location $\left(\mathbf{r}_{i}\right)$ and polarizability $\left(\alpha_{i}\right)$ of $N$-point dipoles are essential parameters for DDA simulation. The dipole moment of targeted geometry is expressed as

$$
\begin{gathered}
\mathbf{p}_{i}=\alpha_{i} \cdot \mathbf{E}_{i, \text { loc }} \\
\mathbf{E}_{i, \text { loc }}=\mathbf{E}_{i, \text { app }}+\mathbf{E}_{i, \text { ind }} \\
\mathbf{E}_{i, \text { app }}=\mathbf{E}_{0} \mathrm{e}^{\mathrm{i}\left(\mathbf{k} \cdot \mathbf{r}_{i}-w t\right)}
\end{gathered}
$$

$$
\mathbf{E}_{i, \text { ind }}=-\sum_{j \neq i}^{N} A_{i j} \cdot \mathbf{p}_{j} \frac{n !}{r !(n-r) !} \frac{n !}{r !(n-r) !}
$$

where $\mathbf{k}$ represents the incident wave vector, $\mathbf{E}_{i, \text { loc }}$ is the local field established by the applied EM field $\mathbf{E}_{i, \text { app }}$ on the array of dipole points, and $\mathbf{E}_{i, \text { ind }}$ is the induced electric field produced by the radiation of all others $(N-1)$ dipoles acting on the $i$ th individual point dipole [28].

$$
\begin{gathered}
A_{i j} \cdot \mathbf{p}_{j}= \\
\frac{\mathrm{e}^{\mathrm{i} k r_{i j}}}{r_{i j}^{3}}\left\{k^{2} r_{i j}\left(r_{i j} \times \mathbf{p}_{j}\right)+\frac{\left(1-\mathrm{i} k r_{i j}\right)}{r_{i j}^{2}} \times\left[r_{i j}^{2} \mathbf{p}_{j}-3 r_{i j}\left(r_{i j} \cdot \mathbf{p}_{j}\right)\right]\right\}
\end{gathered}
$$

And

where

$$
\mathbf{p}_{i}=\alpha_{i} \cdot\left(\mathbf{E}_{i, \mathrm{app}}-\sum_{j=1}^{N} A_{i j} \cdot \mathbf{p}_{j}\right)
$$

$$
\mathbf{r}_{i j}=\left|\mathbf{r}_{i}-\mathbf{r}_{j}\right| \cdot
$$

Once we know each $p_{i}$ of the target geometry, then it is possible to calculate the extinction, absorption and scattering cross-sections as expressed below [29]:

$$
\begin{gathered}
C_{\mathrm{ext}}=\frac{4 \pi k}{\left|\mathbf{E}_{0}\right|^{2}} \sum_{j=1}^{N} \operatorname{Im}\left\{\mathbf{E}_{j, \mathrm{app}}^{*} \cdot \mathbf{p}_{j}\right\} \\
C_{\mathrm{abs}}=\frac{4 \pi k}{\left|\mathbf{E}_{0}\right|^{2}} \sum_{j=1}^{N}\left\{\operatorname{Im}\left[\mathbf{p}_{j} \cdot\left(\alpha_{j}^{-1}\right)^{*} \mathbf{p}_{j}^{*}\right]-\frac{2}{3} k^{3}\left|\mathbf{p}_{j}\right|^{2}\right\} \\
C_{\mathrm{sca}}=C_{\mathrm{ext}}-C_{\mathrm{abs}} .
\end{gathered}
$$

The extinction efficiency is defined as $Q_{\text {ext }}=c_{\text {ext }} / \pi r_{\text {eff }}^{2}$, where $r_{\text {eff }}$ is an effective radius that characterizes the size of the target. Furthermore, the refractive index sensitivity (RIS) $S$ is defined as the ratio of the resonance wavelength $\left(\Delta \lambda_{\text {res }}\right)$ shift to the change in the refractive index of the surrounding medium $\left(\Delta n_{m}\right)$ :

$$
S=\frac{\Delta \lambda_{\mathrm{res}}}{\Delta n_{m}}=\frac{\delta \lambda_{\mathrm{res}}}{\delta n} .
$$

The FOM is defined as

$$
F O M=\frac{S}{F W H M}
$$

where $F W H M$ stands for the full width at the half maximum.

All calculations are carried out by using DDSCAT 7.3, and the target is represented by an 
array of $N$ dipoles. In the current calculation, the number of dipoles used is $\sim 7 \times 10^{4}$ in order to accurately represent the target geometry and for better convergence. The size of the target is characterized by "effective radius" $a_{\text {eff }}=(3 V / 4 \pi)^{1 / 3}$; and $V$ is the actual volume of the material in the target. For triangular prism, the volume is defined as $V=(1 / 2) \cdot(b \cdot h \cdot l)$, where $b, h$, and $l$ are the base, height, and length of a triangular prism. In triangular prism study, the lengths of all sides of the triangular prism are taken equal viz. $a=b=c$ (as shown in Fig. 1), and $h$ is calculated by using Pythagoras theorem (i.e., $h=\sqrt{3} / 2 \cdot a$ ). Therefore, the volume of the triangular prism is $V=(\sqrt{3} / 4) \cdot a^{2} \cdot l$, where $a \cdot a \cdot l$ is taken as aspect ratio $a: a: l:: 1: 1: 2$ and now $V=(\sqrt{3} / 4) \cdot 2 a^{3}$. Thus, $a_{\text {eff }} \equiv$ $(3 V / 4 \pi)^{1 / 3}=(3 \sqrt{3} / 8 \pi)^{1 / 3} \cdot a=0.5913 \cdot a$. Now, our target should have a thickness $a=33.823 \mathrm{~nm}$ in the $x$ direction or long dimension axis, to make the effective radius as $20 \mathrm{~nm}$. Thus, $a_{\text {eff }}$ is considered as $20 \mathrm{~nm}, 30 \mathrm{~nm}, 40 \mathrm{~nm}, 50 \mathrm{~nm}, 60 \mathrm{~nm}$, and $70 \mathrm{~nm}$, and calculations are performed in the wavelength range of $200 \mathrm{~nm}-1200 \mathrm{~nm}$. The choice of triangular prism dimension as (1:1:2) in the current study has been motivated due to the increased surface sensitivities with an enlarged aspect ratio [30]. Furthermore, different refractive indices $(n=1.00,1.33,1.44$, and 1.53 ) of the surrounding medium are considered to represent various environments encountered in biomedical applications.

The behavior of surface plasmons of metal nanostructures can be described on the basis of complex dielectric functions. The values of a complex dielectric constant for considered alloys are taken for individual metal from Johnson and Christy, $[31,32]$, and then the average weight percentage method is used to get the values of effective dielectric functions of alloy nanostructures. The dielectric constant values of two bimetallic alloys with the closed composition as $\mathrm{Ag}_{1-x}-\mathrm{Fe}_{x}$ and
$\mathrm{Au}_{1-x}-\mathrm{Fe}_{x}$ (composition, $x=0.25,0.50,0.75$ ) are calculated using the average weight percentage method. The validation of calculated effective dielectric functions for homogeneous distribution has already been done with experimental values in previously published work [33], thus indicating the importance of the inhomogeneous distribution of composition.

\section{Results and discussion}

The optical properties such as absorption efficiencies of $\mathrm{Ag}-\mathrm{Fe}$ alloys nanoparticles are simulated by using the DDA method. The plasmon wavelength is strongly dependent on the particle size due to the spatial distribution of polarized charges over the surface and charge separation. For the NPs with a size smaller than the wavelength of incident light, the NPs feels a field that is spatially constant but with a time-dependent phase, known as a quasi-static limit. In this approximation/limit, the observed LSPR resonances for small particles are attributed to the excitation of dipolar resonance modes, and larger particles with high multipolar distributions are excited due to the phase retardation effect. Figures 2(a) and 2(b) show the calculated absorption spectra of $20 \mathrm{~nm}$ to $70 \mathrm{~nm}$ sizes for both $\mathrm{Ag}_{0.50}-\mathrm{Fe}_{0.50}$ and $\mathrm{Au}_{0.50}-\mathrm{Fe}_{0.50}$ alloy NPs in the air as the surrounding environment. We have chosen 50:50 alloy composition to achieve desired optical properties with low cost, however, other compositions depending on $\mathrm{Ag}$ or $\mathrm{Au}$ content will also contribute equally towards the tunability of optical properties. Further, we prefer to use $20 \mathrm{~nm}$ as the minimum particle size because the variation in LSPR resonance is very small for the particle size, less than $20 \mathrm{~nm}$. The absorption LSPR peak shifts towards the longer wavelength with an increase in absorption efficiencies, when the nanoparticle size increases. The absorption LSPR spectra are located in the UV-visible region and found in the wavelength range between $347 \mathrm{~nm}-434 \mathrm{~nm}$ and $236 \mathrm{~nm}-406 \mathrm{~nm}$ for Ag-Fe and Au-Fe alloys NPs, 
respectively. Furthermore, the dipole resonance dominants for the particle size, less than or equal to $30 \mathrm{~nm}$, and quadrupole resonance mode appears for the particle size, more than or equal to $40 \mathrm{~nm}$, in Ag-Fe alloy NPs as shown in Fig.2(a). NPs with the size of $20 \mathrm{~nm}$ exhibit a narrow and weak resonance peak with the absorption efficiency nearly to 1.33 and 1.46 for $\mathrm{Ag}-\mathrm{Fe}$ and $\mathrm{Au}-\mathrm{Fe}$, respectively. The absorption efficiency increases close to 3.12 and 2.75 for $\mathrm{Ag}-\mathrm{Fe}$ and $\mathrm{Au}-\mathrm{Fe}$ with an increase in the NPs size, respectively. In comparison, $\mathrm{Au}-\mathrm{Fe}$ alloy multipolar peaks appear for all considered particle sizes, and LSPR peaks shift towards the longer wavelength with an increase in the size.

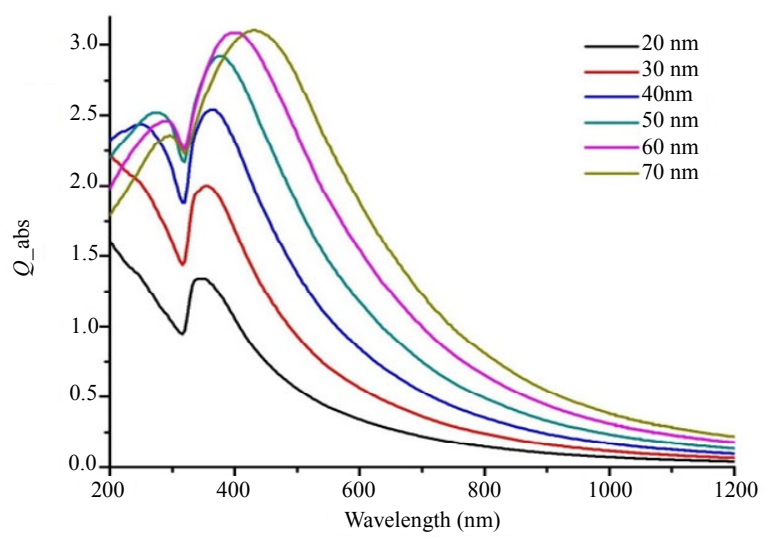

(a)

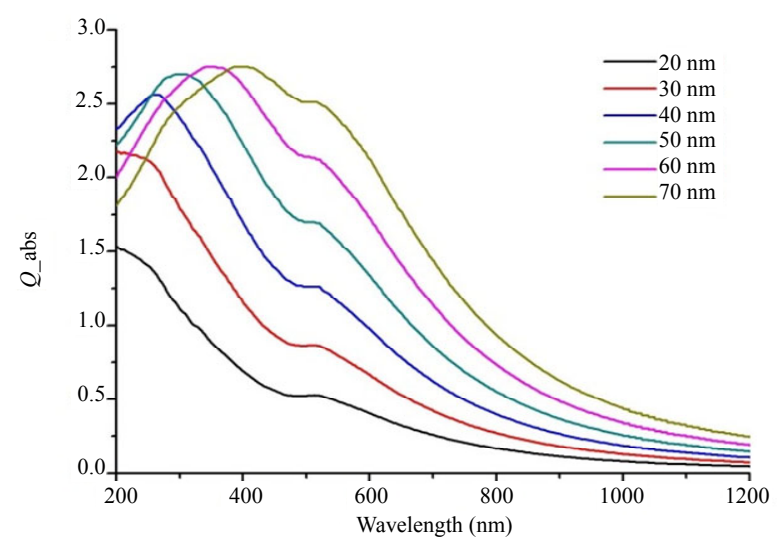

(b)

Fig. 2 Absorption efficiency for (a) $\mathrm{Ag}_{1-x}-\mathrm{Fe}_{x}$ and (b) $\mathrm{Au}_{1-x}-\mathrm{Fe}_{x}$ (composition, $x=0.50$ ) alloy nanoparticles with different sizes.

The optical properties of these alloys are specifically controlled by the composition ratio of silver or gold, whereas, iron contributes towards the magnetic effect for considered magneto-plasmonic alloy NPs. Figures 3 and 4 show the size-dependent relative contributions of absorption and scattering spectra to extinction spectra of both $20 \mathrm{~nm}$ and $70 \mathrm{~nm}$ particle sizes with different compositions of $\mathrm{Ag}_{1-x}-\mathrm{Fe}_{x}$, and $\mathrm{Au}_{1-x}-\mathrm{Fe}_{x}$ (composition, $x=0.25,0.50$, and 0.75 ) bimetallic alloys. The extinction spectra of $20 \mathrm{~nm}$ particle have only absorption contribution, whereas, for the larger size of NPs, extinction spectra contain the contribution of both absorption and scattering as displayed in Figs. 3(d), 3(e), and 3(f), and Figs.4(d), 4(e), and 4(f). An increase in the scattering efficiency for larger NPs sizes can be attributed to the increased surface scattering and appearing of multipole peaks. Furthermore, Ag-Fe alloys NPs show one plasmonic peak whereas two peaks are observed in Au-Fe alloy. In the case of Ag, the d-energy band well below the Fermi energy as compared with $\mathrm{Au}$, and plasmon excitation is more intense with respect to Au. Therefore, the first peak is observed due to LSPR, and the second peak is due to the interband transition in $\mathrm{Au}$, which occurs above $500 \mathrm{~nm}$ wavelength. The interband transitions absorb the energy and affect the intensity and sensitivity to shape. The optical spectra with the symmetrical shape have one plasmonic peak due to dipole resonances. In fact, the formation of the multipolar mode depends on the ways in which the charge density can be polarized. Nanoparticles can be illuminated in the $x-y$ plane representing propagation along the $x$-direction and polarization in the $y$-direction. The orientation of target geometry with respect to the electric field can be considered as $(0,0)$ to $(0,1)$. Literature [34] suggested the maximum absorption efficiency with $(0,1)$ direction (i.e. incidence at $90^{\circ}$ ), and therefore, it is considered in the present study also. Moreover, it is well known that the spherical shape shows a single LSPR peak, and the non-spherical shape may show more than one plasmonic peak, depending on their size, shape, dielectric environment, and symmetry of 
nanostructures, in which they are polarized. Furthermore, the absorption peak intensity gradually increases, while the composition of silver and gold increases in the considered triangular prism nanostructure. The absorption efficiency is a function of NPs volume and increases for certain volume after that scattering will dominate. In the

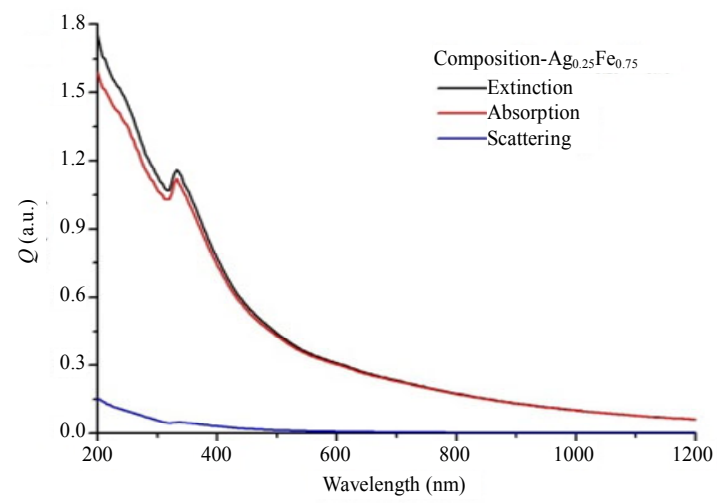

(a)

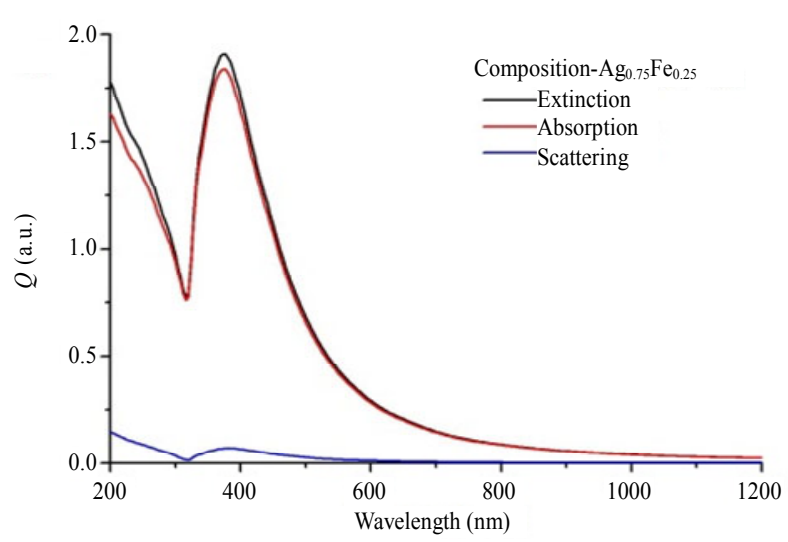

(c)

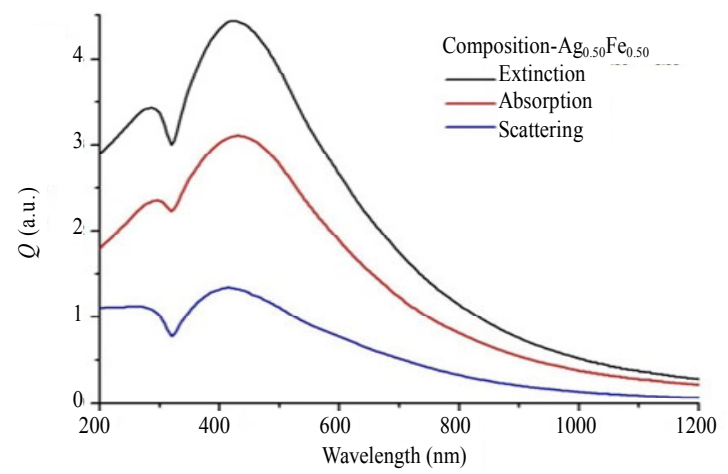

(e) present work on Ag-Fe and Au-Fe alloys, the same trend is observed. As for as [5], the effect of iron content concerned with absorption efficiency decreases with an increase in the iron content in alloy compositions, whereas, the absorption efficiency decreases in the case of coated-Fe NPs [5].

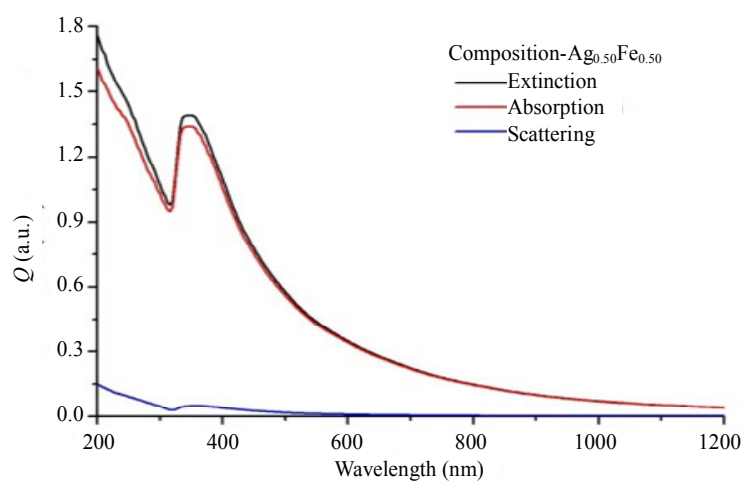

(b)

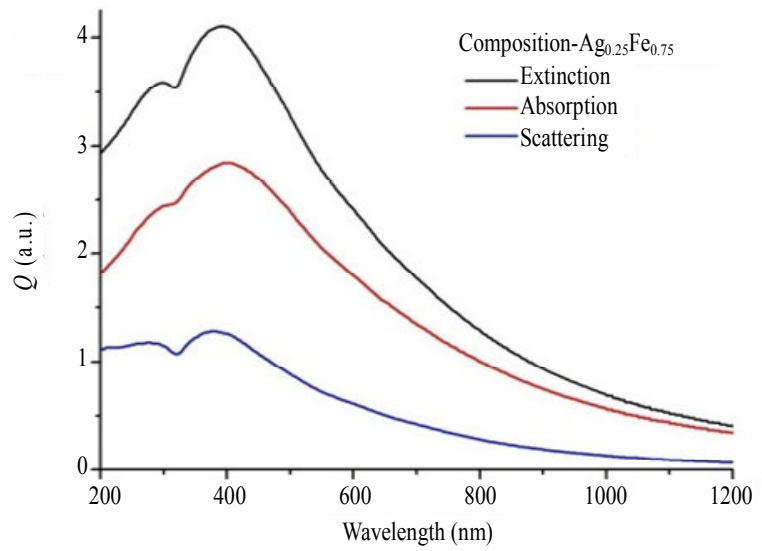

(d)

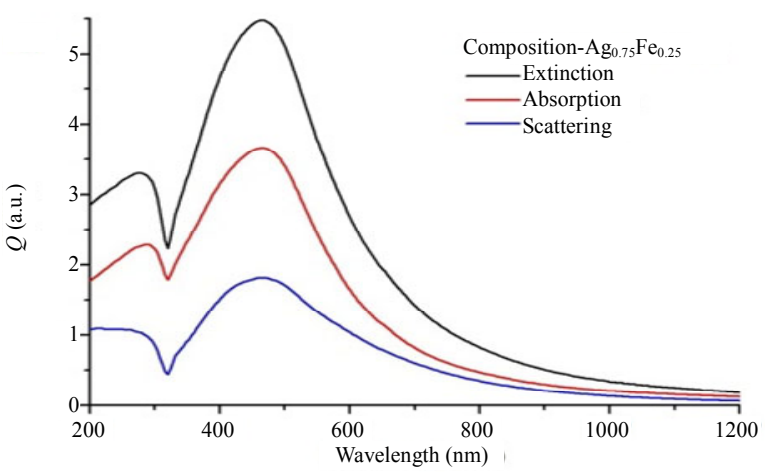

(f)

Fig. 3 Extinction, absorption, and scattering spectra for Ag-Fe bimetallic nanoparticles of different alloy compositions with particle size of $20 \mathrm{~nm}$ in (a), (b), and (c) and particle size of $70 \mathrm{~nm}$ in (d), (e), and (f). 


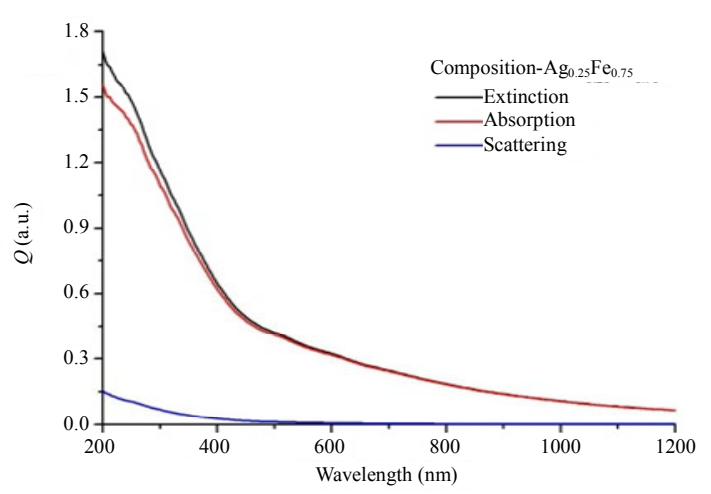

(a)

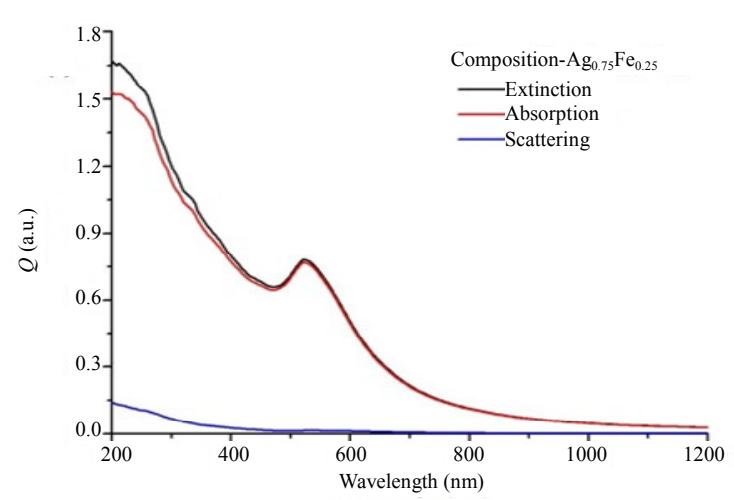

(c)

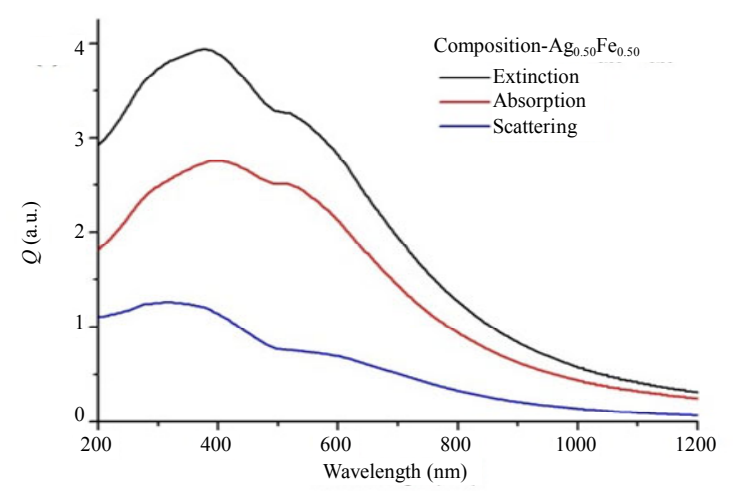

(e)

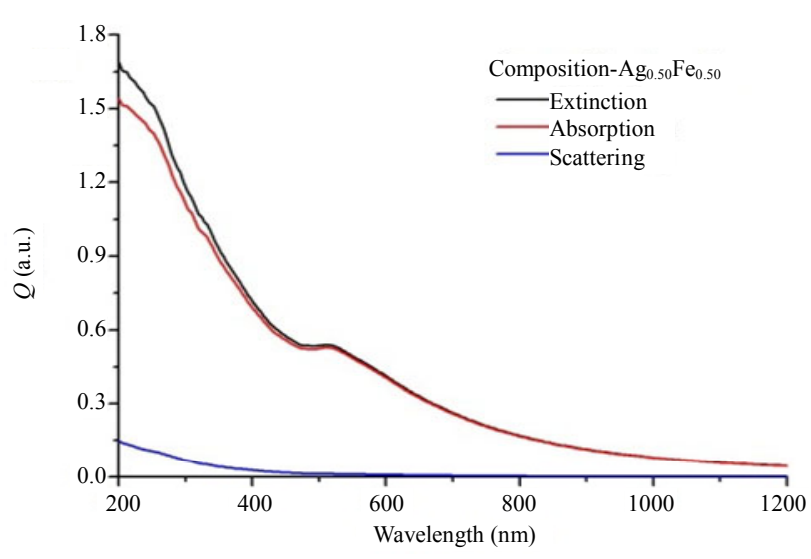

(b)

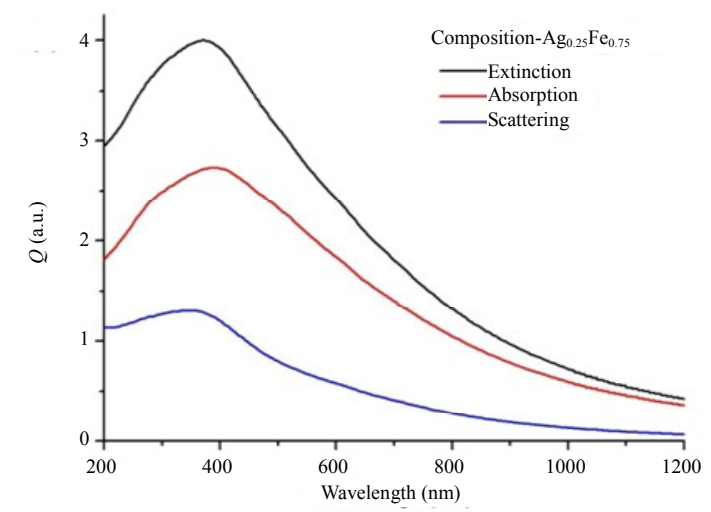

(d)

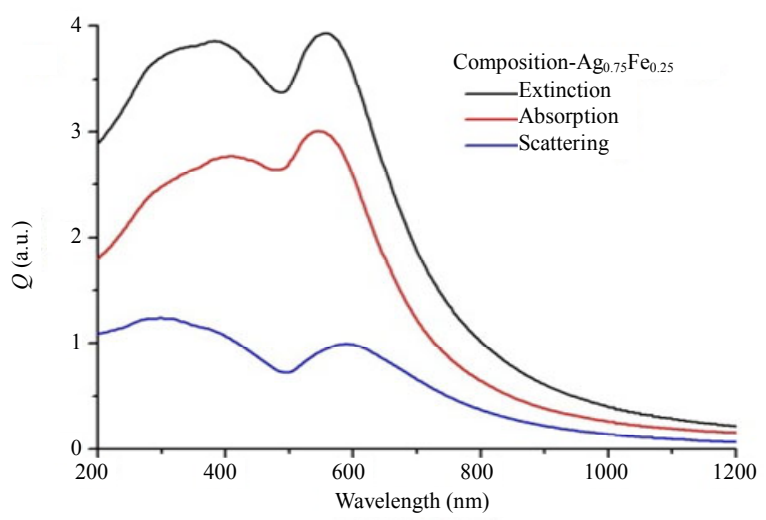

(f)

Fig. 4 Extinction, absorption, and scattering spectra for Au-Fe bimetallic nanoparticles with different alloy compositions with particle size of $20 \mathrm{~nm}$ in (a), (b), and (c) and particle size of in $70 \mathrm{~nm}$ (d), (e), and (f).

All the calculations are initially performed with $7 \times 10^{4}$ dipoles for isolated NPs, but some calculations are repeated with $1 \times 10^{5}$ dipoles to illustrate the reliability of earlier calculations for isolated NPs of $\mathrm{Ag}_{0.50} \mathrm{Fe}_{0.50}$ and are graphically shown in Fig. 5(b). It is very clear from Fig. 5(b) that the variation in the absorption intensity of the LSPR peak does not depend on the number of dipoles. Furthermore, calculations are also performed with rounded corners for the real experimental technologies. Now, we consider $1 \times 10^{4}$ and $1 \times 10^{5}$ dipoles for rounded and sharped corners, respectively. Figure 5 shows the calculated absorption of $20 \mathrm{~nm}$ to $70 \mathrm{~nm}$ sizes for both rounded 
and sharped corners of $\mathrm{Ag}_{0.50}-\mathrm{Fe}_{0.50}$ alloy NPs in the air surrounding environment $(n=1.00)$. We have calculated the FWHM as a function size for rounded and sharped corners with air surrounding medium $(n=1.00)$ shown in Fig. 6 (A- $1 \times 10^{4}$ dipoles, B- $7 \times 10^{4}$ dipoles, C- $1 \times 10^{5}$ dipoles). Since FWHM aids its use in particular applications, e.g., larger FWHM with enhanced scattering efficiency is used in the plasmonic solar cell to increase absorption efficiency, and lower FWHM helps in sensing applications. As the particle size increases from $20 \mathrm{~nm}$ to $70 \mathrm{~nm}$, the values of the FWHM are found

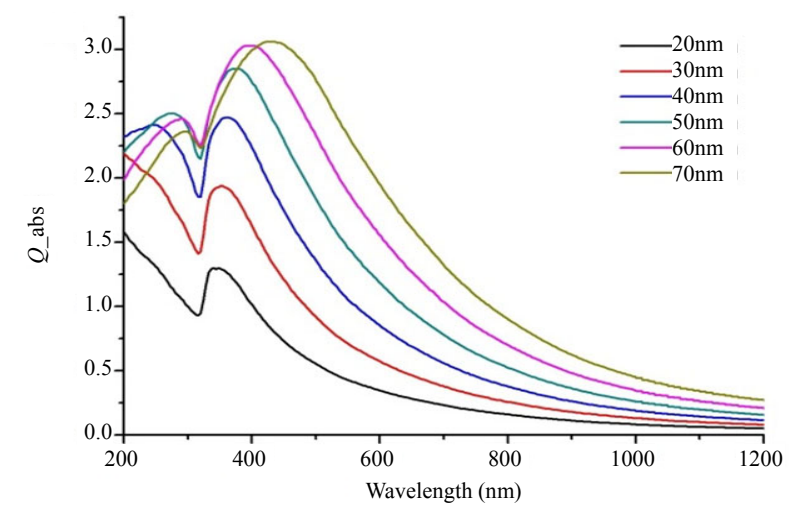

(a) between the range $93 \mathrm{~nm}-222 \mathrm{~nm}$ and $88 \mathrm{~nm}-214 \mathrm{~nm}$ for rounded and sharped corners, respectively. This FWHM is contributed by imaginary parts of the dielectric function. Hence, the sharped corners have a smaller value of the FWHM in comparison with the rounded corners of the triangular prism. Therefore, the FOM [as calculated in (11)] will be higher for NPs with the sharped corners as compared with NPs with the rounded corners. The results that the sharpness is known to induce a higher figure of merit for plasmonic sensing are well supported by other research findings [35].

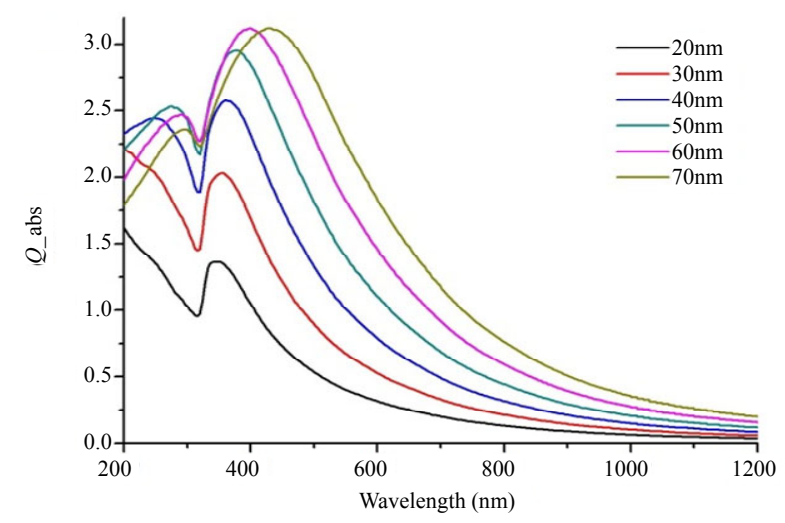

(b)

Fig. 5 Absorption spectra of $\mathrm{Ag}_{0.50}-\mathrm{Fe}_{0.50}$ alloys nanoparticle for the (a) rounded and (b) sharped corners of the triangular prism.

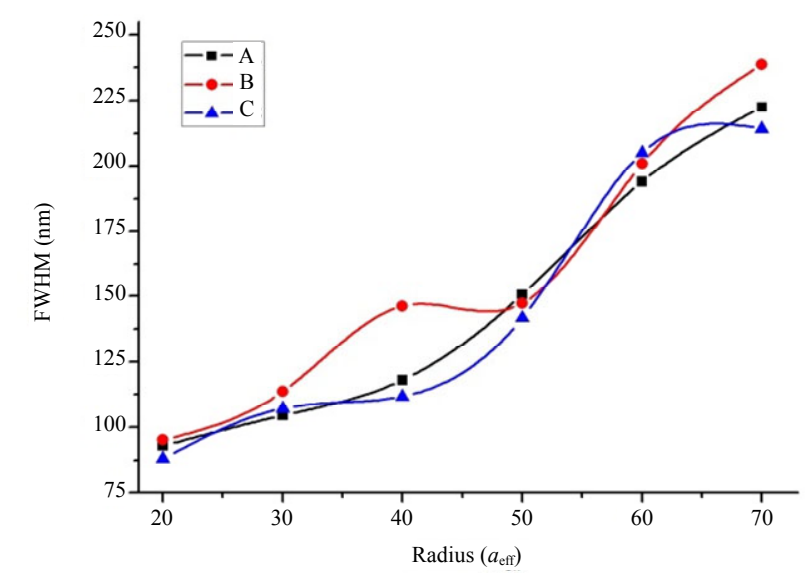

Fig. 6 FWHM as a function of NPs size for $\mathrm{Ag}_{0.50} \mathrm{Fe}_{0.50}$ alloy with different number of dipoles.

The human body contains various fluids (blood or perfused tissue), which can be approximated as aqueous environments. Thus, the optical properties of the considered $\mathrm{Ag}_{0.05}-\mathrm{Fe}_{0.05}$ alloy are calculated in different surrounding environments in order to represent different environments encountered in biomedical applications [36] and also find the favorable and unfavorable effects of considered alloy NPs. The calculated spectra of $20 \mathrm{~nm}, 30 \mathrm{~nm}$, $40 \mathrm{~nm}, 50 \mathrm{~nm}, 60 \mathrm{~nm}$, and $70 \mathrm{~nm}$ particle sizes with the refractive index of the different surrounding media, $n=1.00,1.33,1.44$, and 1.53 , are shown in Fig. 7. When the size of NPs increases from $20 \mathrm{~nm}$ to $70 \mathrm{~nm}$, the resonance wavelength shifts from $347 \mathrm{~nm}$ to $434 \mathrm{~nm}, 381 \mathrm{~nm}$ to $610 \mathrm{~nm}, 388 \mathrm{~nm}$ to $686 \mathrm{~nm}$, and $400 \mathrm{~nm}$ to $727 \mathrm{~nm}$ for $n=1.00,1.33,1.44$, and 1.53 surrounding media, respectively, as shown in Fig. 7(a). As the ambient refractive index increases from 1.00 to 1.53 , a large shift in the LSPR peak has been identified between $347 \mathrm{~nm}$ and $727 \mathrm{~nm}$ wavelengths. Figure 7(b) shows the variation in the 
absorption efficiency, defined as the ratio of the absorption cross section of the particle to the area of the geometrical cross-section of the particle in size. No effective variation in efficiency peaks is observed with the change in the refractive index of

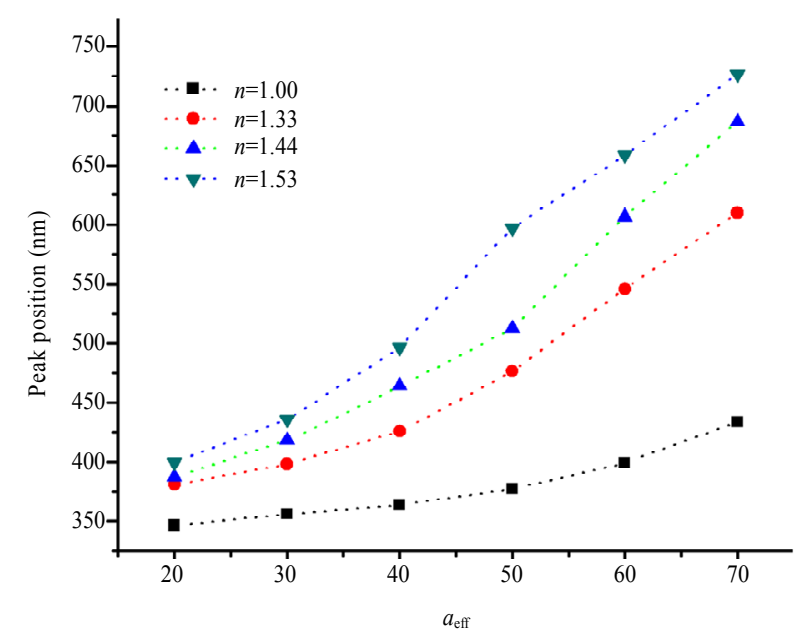

(a) the surrounding medium. But the absorption efficiency linearly increases from 1.33 to $3.10,1.74$ to $3.23,1.80$ to 3.29 , and 1.84 to 3.38 with the surrounding media of $n=1.00,1.33,1.44$, and 1.53 respectively as the particle size increases.

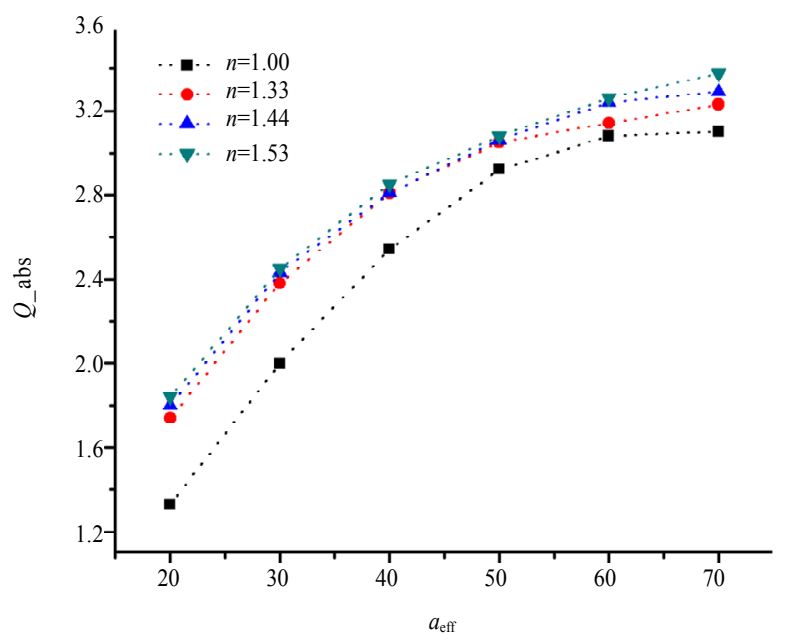

(b)

Fig. 7 Variations in (a) LSPR peak wavelength and (b) absorption efficiency of Ag-Fe nanosphere with size.

Figures 8(a) and 8(b) show the dependence of the dipole LSPR peak position (resonance wavelength) of $\mathrm{Ag}_{0.50} \mathrm{Fe}_{0.50}$ and $\mathrm{Au}_{0.50} \mathrm{Fe}_{0.50}$ alloys for different particle sizes with the refractive index $(n=$ $1.00,1.33,1.44$, and 1.53). When the refractive index of the surrounding medium increases, the linear shift is observed in the plasmon resonance towards the longer wavelength. The nanosensors

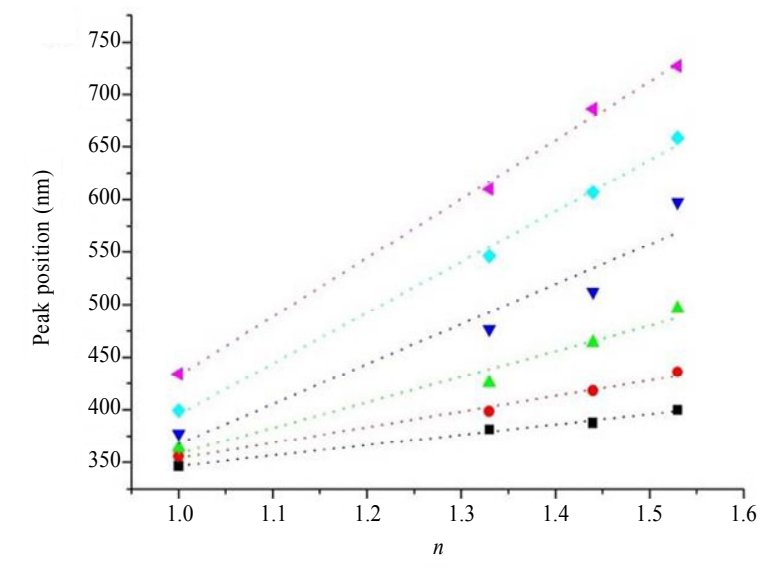

(a) with higher sensitivity $(S)$ and figure of merit (FOM) exhibit better sensing performance, therefore, the refractive index sensitivity is obtained by the above-stated parameter, $S$ [in (10)], and the resonance wavelength is expressed with a refractive index using a linear equation, $S=y n+x$ ( $x$ and $y$ are fitting parameters) given in Tables 1 and 2, respectively.

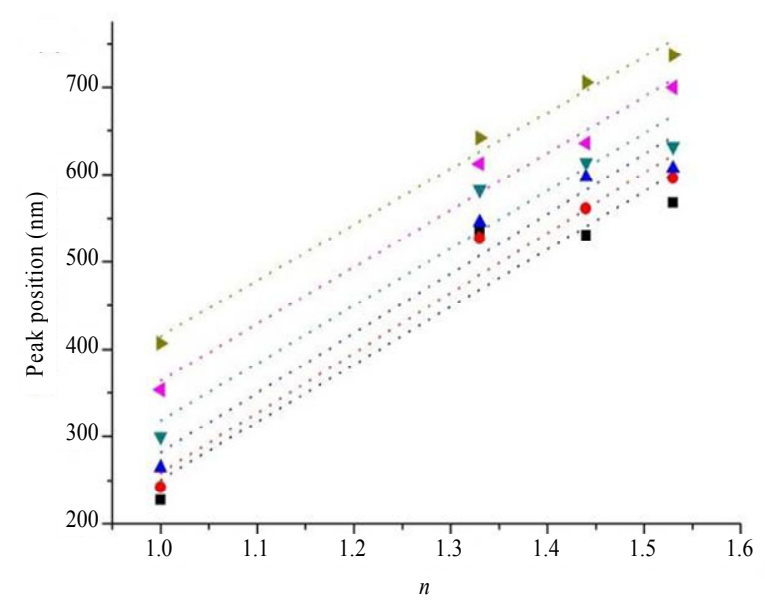

(b)

Fig. 8 Relationship between the resonance wavelength and refractive index of the surrounding medium: (a) Ag-Fe and (b) Au-Fe alloys nanoparticles. 
Table 1 Fitting parameters $x$ and $y$ for $\mathrm{Ag}_{0.50} \mathrm{Fe}_{0.50}$ alloy with different sizes.

\begin{tabular}{ccccc}
\hline \multirow{2}{*}{ Size } & \multicolumn{2}{c}{$x$} & \multicolumn{2}{c}{$y$} \\
\cline { 2 - 5 } & Value & Standard error & Value & Standard error \\
\hline 20 & 248.19 & 7.68 & 98.53 & 5.73 \\
30 & 205.56 & 14.42 & 148.24 & 10.76 \\
40 & 115.59 & 39.55 & 242.86 & 29.51 \\
50 & -12.07 & 96.74 & 379.60 & 72.19 \\
60 & -87.33 & 28.20 & 483.00 & 21.04 \\
70 & -126.12 & 25.64 & 558.70 & 19.13 \\
\hline
\end{tabular}

Table 2 Fitting parameters $x$ and $y$ for $\mathrm{Au}_{0.50} \mathrm{Fe}_{0.50}$ alloy with different sizes.

\begin{tabular}{ccccc}
\hline \multirow{2}{*}{ Size } & \multicolumn{3}{c}{$x$} & \multicolumn{2}{c}{$y$} \\
\cline { 2 - 5 } & Value & Standard error & Value & Standard error \\
\hline 20 & -408.91 & 183.74 & 659.54 & 137.11 \\
30 & -428.79 & 124.25 & 687.03 & 92.72 \\
40 & -401.32 & 136.81 & 682.93 & 102.09 \\
50 & -339.55 & 146.02 & 657.70 & 108.96 \\
60 & -287.43 & 92.01 & 651.24 & 68.66 \\
70 & -225.46 & 62.43 & 640.15 & 46.59 \\
\hline
\end{tabular}

Figure 9 shows the RIS of $\mathrm{Ag}_{0.50} \mathrm{Fe}_{0.50}$ and $\mathrm{Au}_{0.50} \mathrm{Fe}_{0.50}$ alloy systems for the triangular prism with an aspect ratio of the effective radius. The calculated results show that the refractive index sensitivities $(S)$ of both $\mathrm{Ag}-\mathrm{Fe}$ and $\mathrm{Au}-\mathrm{Fe}$ alloy NPs systems for $20 \mathrm{~nm}, 30 \mathrm{~nm}, 40 \mathrm{~nm}, 50 \mathrm{~nm}, 60 \mathrm{~nm}$, and $70 \mathrm{~nm}$ are $99 \mathrm{~nm} / \mathrm{RIU}, 149 \mathrm{~nm} / \mathrm{RIU}, 243 \mathrm{~nm} / \mathrm{RIU}$, $380 \mathrm{~nm} / \mathrm{RIU}, 483 \mathrm{~nm} / \mathrm{RIU}, 559 \mathrm{~nm} / \mathrm{RIU}$, and $660 \mathrm{~nm} / \mathrm{RIU}, 687 \mathrm{~nm} / \mathrm{RIU}, 683 \mathrm{~nm} / \mathrm{RIU}, 658 \mathrm{~nm} / \mathrm{RIU}$, $651 \mathrm{~nm} / \mathrm{RIU}, \quad 640 \mathrm{~nm} / \mathrm{RIU}$, respectively in accordance with the geometric size parameter. Thus, the refractive index sensitivity is higher for $70 \mathrm{~nm}$ and $30 \mathrm{~nm}$ particle sizes of $\mathrm{Ag}-\mathrm{Fe}$ and $\mathrm{Au}-\mathrm{Fe}$ alloys, respectively. A sharp increase in the $S$ value of $\mathrm{Ag}-\mathrm{Fe}$ is observed, as the size of particle increases but for Au-Fe, the refractive index sensitivity value decreases above the $30 \mathrm{~nm}$ particle size. Thus, it is concluded that the sensitivity increases for $\mathrm{Ag}-\mathrm{Fe}$ and decreases for $\mathrm{Au}-\mathrm{Fe}$ as the size $\left(a_{\mathrm{eff}}\right)$ increases.
When the geometry parameter is the same, the refractive index sensitivity of the Au-Fe alloy is higher than that of the Ag-Fe alloy. Therefore, the triangular prism of $\mathrm{Au}-\mathrm{Fe}$ alloy NPs is more suitable for biosensing applications.

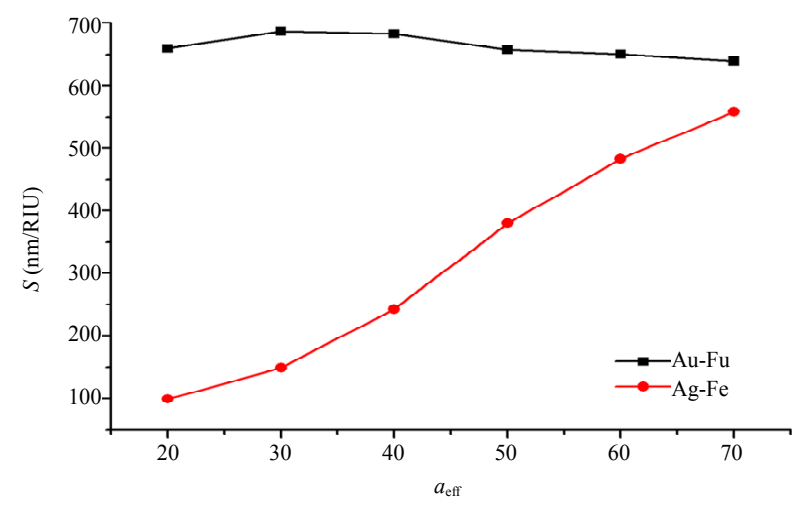

Fig. 9 Relationship between the refractive index sensitivity $(S)$ and the particle size $\left(a_{\text {eff }}\right)$.

The overall performance of LSPR based nanosensors is determined by calculating the FOM. We have calculated the FOM as a function of the size for air $(n=1.00)$ and water $(n=1.33)$ by calculating the FWHM in the same surrounding medium. Figure 10 depicts the FOM with varying the particle size $\left(a_{\text {eff }}\right)$ of Ag-Fe and Au-Fe NPs. In the case of Ag-Fe, with $n=1$, the FOM increases gradually for $20 \mathrm{~nm}$ to $50 \mathrm{~nm}$ in size and then starts decreasing, and with $n=1.33$, the FOM linearly increases above the $30 \mathrm{~nm}$ size. Hence, FOM increases when the particle size increases. In the case of $\mathrm{Au}-\mathrm{Fe}$, with $n=1$, the FOM gradually decreases with an increase in the size, and with $n=$ 1.33 , no change is observed below $40 \mathrm{~nm}$ and above the $50 \mathrm{~nm}$ particle sizes, and only a shift is found in the FOM value between $40 \mathrm{~nm}$ and $50 \mathrm{~nm}$ particle sizes. Moreover, Ag-Fe and Au-Fe alloy NPs exhibit a higher value of FOM for $50 \mathrm{~nm}$ and $20 \mathrm{~nm}$, respectively in the air $(n=1.00)$ surrounding medium. Hence, FOM increases with the value of the refractive index of the surrounding medium, and the effective changes are observed for $n=1.00$ and $n=1.33$ surrounding media. Thus, FOM of Ag-Fe 
increases and decreases for $\mathrm{Au}-\mathrm{Fe} \mathrm{NPs}$ as the size increases because FWHM for gold is more than that of silver NPs under the similar conditions. Our simulation results suggest that Ag-Fe alloy NPs give a higher FOM than the Au-Fe alloy NPs. Hence, it is

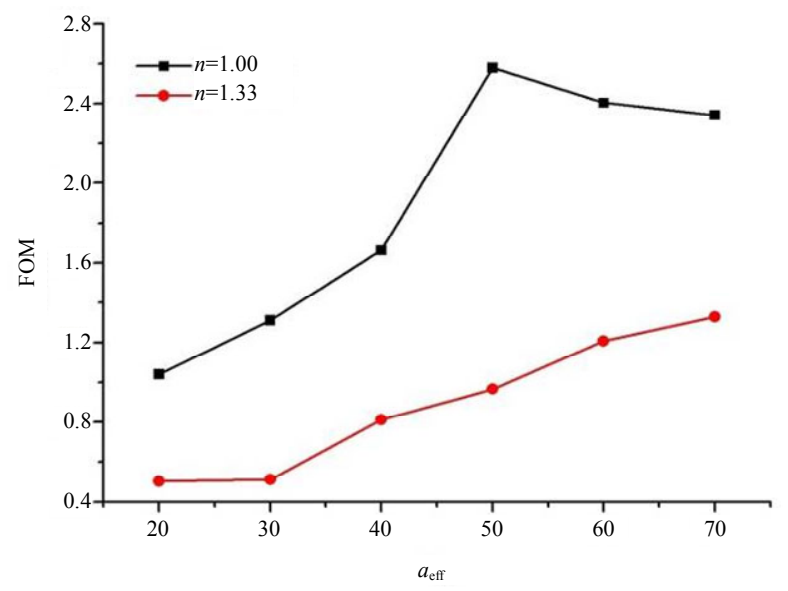

(a) observed that the shift of plasmon resonance towards the longer wavelength and the amount of shift show their dependence on the material, alloy compositions, particle size, and surrounding media.

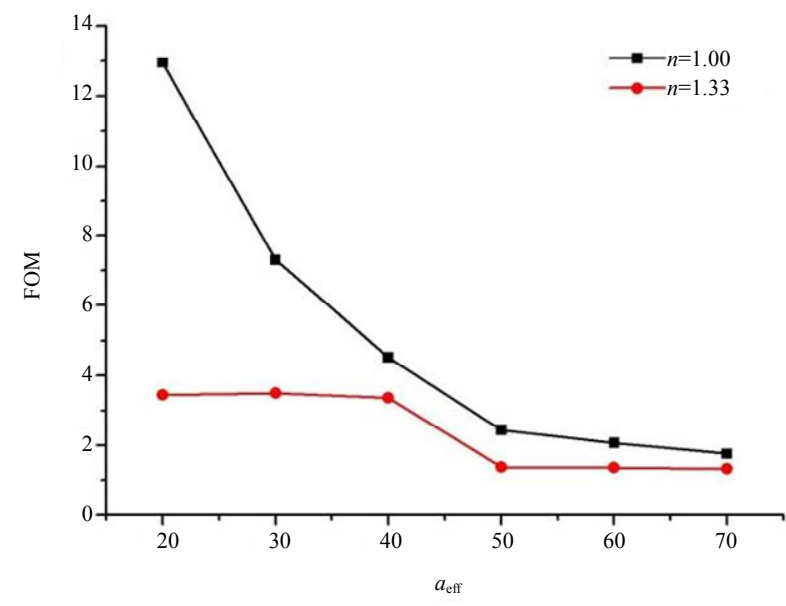

(b)

Fig. 10 Graph between the FOM and the size of (a) $\mathrm{Ag}_{0.50} \mathrm{Fe}_{0.50}$ and (b) $\mathrm{Au}_{0.50} \mathrm{Fe}_{0.50}$ NPs.

\section{Conclusions}

In this work, the refractive index sensitivity properties of the localized surface plasmons for the triangular prism of $\mathrm{Ag}-\mathrm{Fe}$ and $\mathrm{Au}-\mathrm{Fe}$ alloys NPs are simulated by using the DDA method. The effect of the nanoparticle size on the resonance wavelength, sensitivity, and figure of merit are quantitatively analyzed. An increase in the particle size induces the increment of the absorption efficiency value and peak positions towards the longer wavelengths on the ultraviolet and near-infrared regions. The calculated LSPR wavelength shows a high sensitivity to small variations in the refractive index of the surrounding medium. The results reveal that the Ag-Fe alloy has a higher refractive index sensitivity and FOM as compared with the Au-Fe alloy of the triangular prism nanostructure. The optimized size of $70 \mathrm{~nm}$ and $20 \mathrm{~nm}$ of $\mathrm{Ag}-\mathrm{Fe}$ and $\mathrm{Au}-\mathrm{Fe}$ alloys are also investigated for the high refractive index sensitivity and FOM. The size optimization analysis offers a new era with magneto-plasmonic (combination of magnetic and noble metal) NPs in sensing applications.

\section{Acknowledgement}

The author, Pradeep BHATIA, acknowledges B. T. DRAINE and P. J. FLATAU for the use of their code DDSCAT 7.3.

Open Access This article is distributed under the terms of the Creative Commons Attribution 4.0 International License (http://creativecommons.org/licenses/by/4.0/), which permits unrestricted use, distribution, and reproduction in any medium, provided you give appropriate credit to the original author(s) and the source, provide a link to the Creative Commons license, and indicate if changes were made.

\section{References}

[1] I. M. Billas, A. Chatelain, and W. A. de Heer, "Magnetism from the atom to the bulk in iron, cobalt, and nickel clusters," Science, 1994, 265(5179): 1682-1684.

[2] J. Liu, S. Z. Qiao, Q. H. Hu, and G. Q. Lu, "Magnetic nanocomposites with mesoporous structures: synthesis and applications," Small, 2011, 7(4): 
425-443.

[3] E. A. Kwizera, E. Chaffin, Y. Wang, and X. Huang, "Synthesis and properties of magnetic-optical core-shell nanoparticles," RSC Advances, 2017, 7(28): 17137-17153.

[4] L. Lu, W. Zhang, D. Wang, X. Xu, J. Miao, and Y. Jiang, "Fe@Ag core-shell nanoparticles with both sensitive plasmonic properties and tunable magnetism," Materials Letters, 2010, 64(15): 1732-1734.

[5] V. Amendola, R. Saija, O. M. Marago, and M. A. Iati, "Superior plasmon absorption in iron-doped gold nanoparticles," Nanoscale, 2015, 7(19): 8782-8792.

[6] E. A. Chaffin, S. Bhana, R. T. O’Connor, X. Huang, and Y. Wang, "Impact of core dielectric properties on the localized surface plasmonic spectra of gold-coated magnetic core-shell nanoparticles," The Journal of Physical Chemistry B, 2014, 118(49): 14076-14084.

[7] J. Sekhon and S. S. Verma, "Tunable plasmonic properties of silver nanorods for nanosensing applications," Journal of Materials Science, 2012, 47(4): 1930-1937.

[8] J. Zhai and J. Li, "Investigation on the sensitivity and FOM of Ag nanoparticles and nanoarrays," Plasmonics, 2018, https://doi.org/10.1007/s11468018-0842-z.

[9] J. Katyal and R. K. Soni, "Size-and shape-dependent plasmonic properties of aluminum nanoparticles for nanosensing applications," Journal of Modern Optics, 2013, 60(20): 1717-1728.

[10] G. H. Chan, J. Zhao, G. C. Schatz, and R. P. Van Duyne, "Localized surface plasmon resonance spectroscopy of triangular aluminum nanoparticles," The Journal of Physical Chemistry C, 2008, 112(36): 13958-13963.

[11] J. Kelly, G. Keegan, and M. Brennan-Fournet, "Triangular silver nanoparticles: their preparation, functionalization and properties," Acta Physica Polonica A, 2012, 122(2): 337-345.

[12] P. Tuersun, T. Yusufu, A. Yimiti, and A. Sidike, "Refractive index sensitivity analysis of gold nanoparticles," International Journal for Light and Electron Optics, 2017, 149: 384-390.

[13] F. K. Guedje, M. Giloan, M. Potara, M. N. Hounkonnou, and S. Astilean, "Optical properties of single silver triangular nanoprism," Physica Scripta, 2012, 86(5): 055702-055708.

[14] K. L. Kelly, E. Coronado, L. L. Zhao, and G. C. Schatz, "The optical properties of metal nanoparticles: the influence of size, shape, and the dielectric environment," Journal of Physical Chemistry B, 2003, 107(3): 668-677.

[15] Y. Yanase, T. Hiragun, K. Ishii, T. Kawaguchi, T. Yanase, M. Kawai, K. Sakamoto, and M. Hide, "Surface plasmon resonance for cell-based clinical diagnosis," Sensors, 2014, 14(3): 4948-4959.

[16] B. J. Yakes, J. Deeds, K. White, and S. L. DeGrasse,
"Evaluation of surface plasmon resonance biosensors for detection of tetrodotoxin in food matrices and comparison to analytical methods," Journal of Agricultural and Food Chemistry, 2010, 59(3): 839-846.

[17] M. N. Weiss, R. Srivastava, H. Groger, P. Lo, and S. F. Lu, "A theoretical investigation of environmental monitoring using surface plasmon resonance waveguide sensors," Sensors and Actuators A: Physical, 1995, 51(2-3): 211-217.

[18] V. Amendola, R. Pilot, M. Frasconi, O. M. Marago, and M. A. Iati, "Surface plasmon resonance in gold nanoparticles: a review," Journal of Physics: Condensed Matter, 2017, 29(20): 203002.

[19] V. Amendola, S. Scaramuzza, F. Carraro, and E. Cattaruzza, "Formation of alloy nanoparticles by laser ablation of $\mathrm{Au} / \mathrm{Fe}$ multilayer films in liquid environment," Journal of Colloid and Interface Science, 2017, 489: 18-27.

[20] P. Wagener, J. Jakobi, C. Rehbock, V. S. K. Chakravadhanula, C. Thede, U. Wiedwald, and S. Barcikowski, "Solvent-surface interactions control the phase structure in laser-generated iron-gold core-shell nanoparticles," Scientific Reports, 2016, 6: 23352.

[21] S. Scaramuzza, D. Badocco, P. Pastore, D. F. Coral, M. B. Fernandez van Raap, and V. Amendola, "Magnetically assembled SERS substrates composed of iron-silver nanoparticles obtained by laser ablation in liquid," ChemPhysChem, 2017, 18(9): 1026-1034.

[22] Z. Swiatkowska-Warkocka, A. Pyatenko, F. Krok, B. R. Jany, and M. Marszalek, "Synthesis of new metastable nanoalloys of immiscible metals with a pulse laser technique," Scientific Reports, 2015, 5: 9849.

[23] S. S. Verma, P. Bhatia, and M. M. Sinha, "Optical effects of triangular shaped gold-iron nanoparticles," Nano Science \& Nano Technology: An Indian Journal, 2018, 12(1): 123-129.

[24] B. T. Draine and P. J. Flatau, "Discrete-dipole approximation for scattering calculations," Journal of Optical Society of America A, 1994, 11(4): 1491-1499.

[25] S. D. Gedney, "Introduction to the finitedifference-time-domain (FDTD) method for electromagnetics," Synthesis Lectures on Computational Electromagnetics, 2011, 6(1): 1-250.

[26] P. Monk, Finite element methods for Maxwell's equations. Oxford: Oxford University Press, 2003.

[27] B. T. Draine and P. J. Flatau, "Discrete-dipole approximation for periodic targets: theory and tests," Journal of Optical Society of America A, 2008, 25(11): 2693-2703.

[28] B. T. Draine and P. J. Flatau, "User guide for the discrete dipole approximation code DDSCAT 7.3," arXiv preprint arXiv, 2013, 1305.6497. 
[29] C. F. Bohren and D. R. Huffman, Absorption and scattering of light by small particles. New York: John Wiley and Sons, 2008.

[30] C. Y. Tsai, K. H. Chang, C. Y. Wu, and P. T. Lee, "The aspect ratio effect on plasmonic properties and biosensing of bonding mode in gold elliptical nano-ring arrays," Optics Express, 2013, 21(12): 14090-14096.

[31] P. B. Johnson and R. W Christy, "Optical constants of the noble metals," Physical Review B, 1972, 6(12): 4370-4379.

[32] P. B. Johnson and R. W. Christy, "Optical constants of transition metals: $\mathrm{Ti}, \mathrm{V}, \mathrm{Cr}, \mathrm{Mn}, \mathrm{Fe}, \mathrm{Co}, \mathrm{Ni}$, and Pd," Physical Review B, 1974, 9(12): 5056-5070.

[33] P. Bhatia, S. S. Verma, and M. M. Sinha, "Optical properties simulation of magneto-plasmonic alloys nanostructures," Plasmonics, 2018, https://doi.org/ 10.1007/s11468-018-0839-7.

[34] M. Alsawafta, M. Wahbeh, and V. V. Truong, "Simulated optical properties of gold nanocubes and nanobars by discrete dipole approximation," Journal of Nanomaterials, 2012, 2012: 1-9.

[35] K. M. Mayer and J. H. Hafner, "Localized surface plasmon resonance sensors," Chemical Reviews, 2011, 111(6): 3828-3857.

[36] T. Boothe, L. Hilbert, M. Heide, L. Berninger, W. B. Huttner, V. Zaburdaev, et al., "A tunable refractive index matching medium for live imaging cells, tissues and model organisms," eLife, 2017, 6: 27240.001-27240.013. 